\author{
Ajda Gabrič \\ Doktorandin der Philosophischen Fakultät, \\ Universität Ljubljana \\ Slowenien \\ ajda.gabric@gmail.com
}

UDK 811.112.2'243:374.7

DOI: $10.4312 /$ vestnik.11.173-190

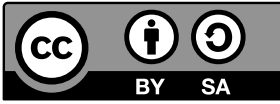

\title{
ANALYSE DER FEHLER IN TEXTEN DER DEUTSCHLERNENDEN AUF DER ANFÄNGERSTUFE
}

\section{EINLEITUNG}

Im vorliegenden Beitrag werden einige typische Fehler analysiert, die die slowenischen MuttersprachlerInnen beim Verfassen deutscher Texte machen. Mlakar Gračner definiert Fehler in Anlehnung an Lewandowski als „eine Abweichung von geltenden Normen, ein[en] Verstoß gegen sprachliche Richtigkeit, Regelhaftigkeit oder Angemessenheit“" (Lewandowski 1994, zitiert nach Mlakar Gračner 2018: 220).

Der Fehler spielt heutzutage in der fremdsprachlichen Didaktik ,eine zentrale und positive Rolle.“ (Kleppin 1998: 51) In der moderneren Fachliteratur hat sich eine positive Einstellung gegenüber Fehlern durchgesetzt, insbesondere als Konsequenz von Corders Feststellung, dass Fehler ein Beweis der Lernstrategien bei den Lernenden seien. Fehler seien ein natürlicher Bestandteil des Spracherwerbs und ein Beweis des jeweiligen Zustands bei den Lernenden; sie können als Illustration des Fortschritts bzw. des Rückgangs dienen (Muster 2000a: 17). Es wird angenommen, dass Fehler ein notwendiges Vorkommnis beim Erlernen der Sprache sind:

Man geht davon aus, dass [der Fehler] beim kreativen Prozess der Aneignung einer Fremdsprache unumgänglich ist, ja, dass er diesen Prozess sogar widerspiegelt und man eben an einigen Fehlern besonders gut erkennen kann, was eventuell im Gehirn des Lernenden vor sich geht, vielleicht sogar, welche Lernschwierigkeiten auftreten. (Kleppin 1998: 51-52)

Eine Klassifizierung und Analyse der Fehler ist sowohl für die Lehrkräfte, die ihren Unterricht mithilfe dieses Wissens erfolgreicher gestalten und die Ergebnisse besser bewerten können, als auch für die Lernenden, die ihre eigenen Fehler erkennen können, 
sinnvoll (Kleppin 1998: 41). Fehler beim Sprachunterricht können unterschiedlich klassifiziert werden; im vorliegenden Beitrag werden sie nach sprachwissenschaftlichen Kriterien klassifiziert - sie werden in Gruppen unterteilt, die den sprachlichen Ebenen entsprechen. Muster (2000a: 27) führt die folgenden Ebenen an, auf denen Fehler vorkommen:

- Rechtschreibung

- Orthoepie bzw. Rechtlautung

- Morphologie

- Syntax

- Semantik

Andere Autorinnen klassifizieren die sprachlichen Ebenen ähnlich. Bei Kleppin (1998: 42) wird die phonetische/phonologische Ebene aufgelistet, die die Aussprache und die Orthographie fasst. Die Ebenen der Morphologie und der Syntax werden unter zusammen dem Namen „morphosyntaktische Fehler“ (1998: 42) zusammengefasst; der Kategorisierung werden noch die pragmatische und die inhaltliche Ebene hinzugefügt (ebd., 43). Auch bei Mlakar Gračner werden ähnliche Kategorien wie bei Muster aufgelistet, wobei die semantische Ebene weiter gegliedert wird: Der lexikosemantischen Ebene werden so wie bei Kleppin noch die pragmatische und die inhaltliche Ebene hinzugefügt (Mlakar Gračner 2018: 221-222). Die meisten Fehler auf der lexikalischen Ebene „entspringen wortwörtlicher Übersetzung aus dem Slowenischen oder falschem Wörterbuchgebrauch“" (Muster 2000b: 478). Inhaltliche Fehler sind solche, bei denen die sprachliche Äußerung semantisch nicht mit der außersprachlichen Realität übereinstimmt. Schließlich sind die pragmatischen Fehler diejenigen, die „oft durch kulturelle Unterschiede oder Missverständnisse“ entstehen (Mlakar Gračner 2018: 222). Sie zeigen sich als Einhaltung anderer Kommunikationsnormen bzw. sprachliche Handlungen, die in der Fremdsprache ungeeignet sind. Muster führt als typische Beispiele die ungeeigneten Anredeformen, besonders die falschen Pronomen für das Siezen und Duzen, sowie das Verkennen der Unterschiede zwischen der österreichischen und der deutschen Norm und die falschen Gruß- und Anredeformeln an (Muster 2000b: 478).

\section{3}

\section{FEHLERURSACHEN}

Die Ursachen für die Sprachfehler existieren auf mehreren Ebenen. Die erste Gruppe der Ursachen stammt von den Eigenschaften der im Unterricht tätigen Personen - der Lehrer/ Lehrerinnen und der Schüler/Schülerinnen. Bei den Lernenden spielen eine Rolle ihre Motivation, ihre Vorkenntnisse, ihre Kenntnisse anderer Sprachen, ihr Alter, ihr psychischer Zustand (Stress, Müdigkeit, Angst) usw. Auf der Seite der LehrerInnen der Fremdsprache spielt eine wichtige Rolle die Frage, ob der Lehrer/die Lehrerin eine sprachliche Besonderheit, die zu häufigen Fehlern führen kann, genügend erklärt. Die Lehrperson 
muss klar genug hervorheben, wo Fehler in der Fremdsprache vorkommen können und wie man sie vermeiden kann. Dazu gehören genügend Beispiele und auch Übungen zur Wiederholung und zur Durchsetzung einer Struktur.

Ursachen für Fehler stammen auch aus den Unterschieden zwischen den schon bekannten und der zu erlernenden Sprache; sehr wichtig sind hier die Begriffe des Transfers und der Interferenz. Der Transfer ist die „Übertragung von sprachlichen Besonderheiten der Muttersprache auf die Fremdsprache“ (Bußmann 1990: 799). Einfacher gesagt, die schon bekannte Sprache beeinflusst das Lernen und die Performanz in der Fremdsprache, die gelernt wird. Der Transfer kann in unterschiedlichen Situationen unterschiedlich intensiv sein und kann entweder positive oder negative Konsequenzen haben. Der negative Transfer bedeutet die Übertragung der sprachlichen Regeln aus einer Sprache in die andere (die Zielsprache), wobei diese Regeln für die Zielsprache nicht gelten. Als solcher hemmt der Transfer das Lernen, denn er ruft Fehler hervor. Andererseits helfen die Ähnlichkeiten zwischen den Sprachen beim Lernen und beschleunigen den Fremdsprachenerwerb - das ist der positive Transfer. Der negative Transfer ist einfacher zu beobachten als der positive, denn nur der negative führt zu auffallenden sprachlichen Abweichungen (Čok 1999: 51).

Die sprachliche Interferenz zwischen der Erstsprache der Lernenden und der Zielsprache ist ein anderer Name für den negativen Transfer. Sie wird in diesem Fall als die „Beeinflussung eines Sprachsystems durch ein anderes [...] im Individuum“ (Bußmann 1990: 349) verstanden, die folgend eine „Fehlerquelle“ darstellen kann (ebd.). Muster (2000a: 25) meint sogar, dass die Interferenz die häufigste Ursache der sprachlichen Fehler ist. Die Interferenz tritt vor allem in Fällen auf, wo die Erstsprache und die Fremdsprache stark unterschiedlich sind. Muster (2000a: 26) schreibt, dass die Interferenz auf allen Sprachebenen mithilfe der kontrastiven Analyse erklärt werden kann. Die kontrastive Analyse dient auch zur Prognostizierung der Fehler (Muster 2000a: 26). Čok (1999: 49) betont, dass nicht nur die Muttersprache, sondern auch die anderen Fremdsprachen den Erwerb einer neuen Sprache beeinflussen. Muster (2008: 613-614) listet einige fehlerhafte Beispiele aus Schüleraufsätzen auf, die auf den Einfluss der Englischkenntnisse auf den Deutscherwerb verweisen.

Die Interferenz funktioniert auf allen sprachlichen Ebenen, von der phonetischen bis zur syntaktischen. Auf der morphosyntaktischen Ebene ist wenig Platz für Interferenzen, da die Grammatik ein strukturiertes, geschlossenes System ist, das wenig Freiraum zulässt. Die Fehler auf dieser Ebene können wegen Verallgemeinerungen und Vereinfachungen der Regeln entstehen (Čok 1999: 50-51). Fehler als Folge der sprachlichen Interferenz gibt es auch auf der semantischen Ebene, vor allem, wenn Wörter oder sprachliche Strukturen wortwörtlich aus der Muttersprache in die Fremdsprache übersetzt werden. Die Interferenz ist der zugrundeliegende Mechanismus der sogenannten falschen Freunde (ebd., 51), der scheinbar ähnlichen Wörter in verschiedenen Sprachen mit unterschiedlicher Bedeutung. Fehler können aber auch als Folge der ungeschickten Verwendung der 
zweisprachigen Wörterbücher entstehen - die Schüler und Schülerinnen wählen von mehreren angebotenen Übersetzungen eines Worts die falsche aus (Muster 2000a: 59).

Neben der Interferenz zwischen unterschiedlichen Sprachen gibt es auch die sogenannte intralinguale oder innersprachliche Interferenz, bei der die Lernenden eine Struktur bzw. eine sprachliche Regel verwenden, die sie beherrschen, auch wenn es nicht angebracht ist. Entweder verallgemeinern sie die Regeln oder sie vermischen verschiedene sprachliche Regeln, was zu einer fehlerhaften Struktur führt (Muster 2000a: 30-31). Ein schon gemachter Fehler kann auch zu anderen Fehlern innerhalb desselben Satzes führen (ebd., 36).

Vor allem die besseren Lernenden versuchen immer wieder anspruchsvollere Strukturen zu formulieren als diejenigen, die man beim Unterricht gelernt und geübt hat. Dabei setzen sie die sprachlichen Regeln ein, die sie schon kennen, und übertragen sie auch auf unbekannte Formulierungen. Dies zeigt meistens, dass sie schon einen Schritt nach vorne denken und kreativ mit der Sprache umgehen möchten. Deshalb sind solche Versuche von der Lehrperson als positiv zu nehmen, obwohl dabei Fehler gemacht werden.

Im Folgenden werden Fehler aus 40 schriftlichen Aufgaben der erwachsenen Deutschlernenden analysiert. Die Aufsätze wurden von Teilnehmenden in den Kursen auf der Stufe A1 und am Anfang der Stufe A2 des Gemeinsamen Europäischen Referenzrahmens, die Deutsch mithilfe der Lehrwerke studio 21 Al und studio $21 A 2^{l}$ lernen, geschrieben. Die Lernenden hatten vor dem Beginn des Kurses zumeist keine Vorkenntnisse. Vor dem Schreiben der Texte, die teilweise als Hausaufgaben und teilweise als Teil der schriftlichen Prüfungen verfasst wurden, bekamen sie detaillierte Anleitungen mit Stichpunkten, über die sie schreiben sollten. Der Wortschatz zu einzelnen Themen und die typischen Formulierungen, die sie zu verwenden hatten, wurden zuvor im Kurs gelernt und geübt.

Die Stufe A1 ist die niedrigste Stufe, die der GER definiert. Für die Lernenden auf dieser Stufe gilt, dass sie ,vertraute, alltägliche Ausdrücke und ganz einfache Sätze verstehen und verwenden“ können. Sie können „sich und andere vorstellen und anderen Leuten Fragen zu ihrer Person stellen“ und ,auf Fragen dieser Art Antwort geben.“(GER 2013: 35) Nach den Bestimmungen des GER gilt, dass die Lernenden auf dieser Stufe nur „einfache, isolierte Wendungen und Sätze“ schreiben können (GER 2013: 67), was eigentlich bedeutet, dass die AutorInnen der analysierten Aufsätze höhere Schreibkompetenzen haben als erwartet - jedoch basieren ihre Texte meist auf sich wiederholenden, auswendig gelernten Formulierungen.

1 Hermann Funk/Christina Kuhn: studio 21 A1. Berlin: Cornelsen, 2015. Hermann Funk/Christina Kuhn/Britta Winzer Kiontke: studio 21 A2. Berlin: Cornelsen, 2015. 
Diese Lernenden haben also noch relativ niedrige Deutschkenntnisse und es ist zu erwarten, dass sie viele Fehler machen, die die Grundregeln der Sprache verletzen. Sie haben über folgende Themen geschrieben:

- Meine Arbeit (11 Texte)

- Mein Urlaub (13 Texte)

- $\quad$ Mein Tag (3 Texte)

- Dialog: Wir verabreden einen Termin (4 Texte)

- Meine Sprachen- und Lernbiografie $(9 \text { Texte })^{2}$

Die Analyse folgt methodologisch der Fehleranalyse von Doris Mlakar Gračner (2018: 224-225). Fehler werden nach Sprachebenen klassifiziert - die Rechtschreibfehler, die morphologischen, die syntaktischen sowie die lexikalischen und semantischen Fehler konnten berücksichtigt werden. Die Ebene der Aussprache wurde ausgeklammert, da nur schriftliche Texte analysiert wurden. Im Prinzip habe ich mich entschieden, alle Fehler in die folgende Statistik aufzunehmen - im Beispiel wie *Meine aufhgabe sind technise Probleme zu lösen, Projektmanagement machen $(04)^{3}$ bedeutet das, dass das Wort *aufhgabe mehrere Fehlerbeispiele darstellt: Erstens ist es als Nomen fehlerhaft klein geschrieben, zweitens beinhaltet es einen Buchstaben zu viel $(h)$ und drittens steht es im Singular statt im Plural. Ich glaube, dass eine solche Methode sinnvoll ist, denn sie verdeutlicht die einzelnen Fehler. In Fällen, bei denen ein identischer Fehler mehrmals in ein- und demselben Text gemacht wurde, wurden alle Beispiele einzeln gezählt, um die Häufigkeit des Fehlers zu illustrieren.

Es ist wichtig, nochmal zu betonen, dass die meisten Fehler nicht eindeutig klassifiziert werden können. Es ist nicht immer klar, woher ein Fehler stammt. Auch Doris Mlakar Gračner begegnete bei ihrer Klassifizierung dem Problem der eindeutigen Identifizierung der Fehlerursachen und entschied: „Die in der Untersuchung beschriebenen Erklärungen sind dementsprechend als Vermutungen zu betrachten.“ (Mlakar Gračner 2018: 225) Dieser Hinweis gilt auch für die Ergebnisse meiner Analyse.

2 Die Aufsätze zum Thema »Meine Sprachen- und Lernbiografie« beziehen sich auf das Thema, das im ersten Kapitel des Bandes studio 21 A2 behandelt wird. Die Lernenden sind auf fast derselben Stufe wie die Lernenden auf der Stufe A1.

3 Die Nummer in Klammern hinter jedem Zitat aus den Aufsätzen bezieht sich auf den Text, aus dem das Zitat stammt. 
Tabelle 1: Anzahl der Fehler nach sprachlichen Ebenen

\begin{tabular}{|l|l|}
\hline Rechtschreibung & $\mathbf{2 2 1}$ \\
\hline Groß- und Kleinschreibung & 70 \\
\hline Getrennt- und Zusammenschreibung & 10 \\
\hline Buchstabieren & 110 \\
\hline Zeichensetzung & 31 \\
\hline Morphologie & $\mathbf{2 4 2}$ \\
\hline Verb & 61 \\
\hline Nomen & 86 \\
\hline Artikel & 36 \\
\hline Adjektiv & 22 \\
\hline Präposition & 37 \\
\hline Syntax & $\mathbf{5 6}$ \\
\hline Verb im Hauptsatz an falscher Stelle & 22 \\
\hline Verb im Nebensatz an falscher Stelle & 7 \\
\hline Fehlendes Satzglied & 6 \\
\hline Überflüssiges Wort & 14 \\
\hline Anderes & 7 \\
\hline Lexik und Semantik & $\mathbf{4 8}$ \\
\hline Unpassendes Wort; Wort aus einer anderen Sprache verwendet & 42 \\
\hline Unlogische Wortbildung & 1 \\
\hline Falsche Verwendung vom Siezen und Duzen & 2 \\
\hline Falsche Verwendung der Personalpronomen & 3 \\
\hline Textstruktur & $\mathbf{6}$ \\
\hline
\end{tabular}

\subsection{Rechtschreibfehler}

Die deutsche Rechtschreibung stellt, wie aus den Ergebnissen der Analyse folgt, für die Lernenden auf der Anfängerstufe ein großes Problem dar. Problematisch ist sowohl die Setzung der großen und kleinen Anfangsbuchstaben als auch das korrekte Buchstabieren der Wörter. Einige Fehler gab es noch im Bereich der Getrennt- und Zusammenschreibung und der Zeichensetzung.

Die Lernenden begegnen der Besonderheit des Deutschen, dass alle Nomen großgeschrieben werden, schon am Anfang der Sprachkurse, brauchen aber einige Zeit, bevor sie sich daran angewöhnen, automatisch alle Substantive mit einem großen Anfangsbuchstaben zu versehen. Folglich stellen die klein geschriebenen Nomen einen großen Anteil der Rechtschreibfehler dar. Augenfällig sind die Aufsätze 04, 06 und 22, die die 
meisten kleingeschriebenen Substantive beinhalten. Allein im Text 06 sind 9 solche Beispiele zu finden - andererseits werden nur 5 Nomen korrekterweise großgeschrieben, was davon zeugt, dass der Autor dieses Aufsatzes die Rechtschreibregeln noch nicht automatisiert hat.

Überraschend ist die falsche Schreibweise des Pronomens ich, und zwar mit dem großen $I$. Es kann nur angenommen werden, dass dieses Phänomen ein Resultat der Interferenz mit dem Englischen darstellt, da in dieser Sprache das Pronomen $I$ stets großgeschrieben wird. Personalpronomen für andere Personen sind in dieser Hinsicht nicht problematisch. Ein Beispiel dieser Problematik wäre *Kinder haben jeden Abend Eis gewollt. Und Ich auch. (28)

Beispiele, bei denen andere Wortarten fälschlicherweise großgeschrieben werden, sind statistisch nicht relevant, da sie kaum vorkommen. Es kann dabei um Fälle gehen, bei denen andere Wortarten fälschlicherweise als Nomen empfunden werden, weil sie z.B. gleich wie ein Nomen lauten: *Nein, Morgen kann ich leider nicht. (18).

Tabelle 2: Fehler auf der Ebene der Groß- und Kleinschreibung

\begin{tabular}{|l|l|}
\hline Fehler auf der Ebene der Groß- und Kleinschreibung & $\mathbf{7 0}$ \\
\hline Nomen wird kleingeschrieben & 55 \\
\hline Pronomen ich wird großgeschrieben & 7 \\
\hline Andere Wortarten werden großgeschrieben & 7 \\
\hline Anfangsbuchstabe im Satz wird kleingeschrieben & 1 \\
\hline
\end{tabular}

Fehler bei der Zusammenschreibung der Wörter kommen vor allem dann vor, wo im Slowenischen zwei Wörter (meist Adjektiv und Substantiv) für etwas verwendet werden, wofür es im Deutschen eine Zusammensetzung gibt. Es geht also um Interferenz mit dem Slowenischen, z.B. *Schicht Arbeit. (01) - slow. izmensko delo; *Sprach Schule (32) slow. jezikovna šola.

In 2 Beispielen werden Wörter zusammen geschrieben, die getrennt sein müssten, nämlich *Branderburgertör (02) und *zuhause (36). Hier handelt es sich wahrscheinlich um die innersprachliche Interferenz, da die Lernenden die Zusammenschreibung einiger Wörter lernten und sie auf andere übertrugen.

Tabelle 3: Fehler auf der Ebene der Getrennt- und Zusammenschreibung

\begin{tabular}{|l|l|}
\hline Fehler auf der Ebene der Getrennt- und Zusammenschreibung & $\mathbf{1 0}$ \\
\hline Ein Wort wird in zwei Wörter geteilt & 7 \\
\hline Zwei Wörter werden als eins geschrieben & 2 \\
\hline Bindestrich fehlt & 1 \\
\hline
\end{tabular}


Letztlich gibt es auf der Rechtschreibebene noch fehlende Buchstaben, überflüssige Buchstaben sowie falsch ausgewählte Buchstaben mitsamt der Umlaute. Die Lernenden auf der Anfängerstufe haben damit, wie aus meiner Statistik ersichtlich, die meisten Probleme - oder, von der Sicht der Lernstrategien betrachtet, finden sie extrem viele Möglichkeiten, um ein Wort schriftlich wiederzugeben. Eine eindeutige Klassifikation, die alle Fehlerbeispiele systematisch erklären könnte, lässt sich nicht erstellen, da diese Fehler höchst unsystemisch sind. Einige auffällige Regelmäßigkeiten lassen sich jedoch identifizieren:

- Umlaute wurden in 14 Fällen „vergessen“, z.B. *taglich (28). Einen Teil dieser Fälle stellen das Wort für, bei dem der Umlaut viermal ausgelassen wurde (davon dreimal im Text 35), und das Wort Grüße (5 fehlerhafte Beispiele, viermal ohne Umlaut und einmal als Große (04) geschrieben) dar.

- Bei den Konsonantengruppen sch und tsch für das Wiedergeben der Laute, die im Slowenischen mit $\check{S}$ oder $\check{C}$ geschrieben werden, fehlt in 9 Beispielen der eine oder der andere Buchstabe: *Chüss! (02), *Deutshunterricht (33), *English (35). Vor allem aus den Fehlern im Wort Englisch ist es ersichtlich, dass hier auch die Interferenz mit dem Englischen eine wichtige Rolle spielt. Im Text 35 wurde das Wort sechsmal verwendet, davon wurde es zweimal korrekt geschrieben und viermal fehlt ihm das $C$.

- Wahrscheinlich kommt aus dem Englischen auch die Tendenz, dass der Laut [k] mit einem $C$ geschrieben wird, vor allem bei Internationalismen (6 Beispiele): ${ }^{*}$ Caffe (04), *America (21).

- Der Buchstabe $C$ wird auch in 5 Fällen falsch eingesetzt, wenn es um das Wiedergeben des Lautes [ts] geht, der im Slowenischen immer mit einem $C$ geschrieben wird: *Cirkus (06), *Centrum (20). Hier geht es um die Interferenz mit dem Slowenischen.

- Das Wort sehr kommt dreimal falsch geschrieben vor: Als *zer (04) und *zehr (06, 21, wobei diese beiden Texte von demselben Autor stammen). Der Laut $[z]$ wird also mit einem $Z$ wiedergegeben, wie es im Slowenischen üblich ist. Ein ähnliches Beispiel stellt das Wort *Pauze (09) dar.

\subsection{Morphologische Fehler}

Wie aus dem Überblick in der Tabelle 1 ersichtlich, haben die Lernenden die meisten Probleme mit der deutschen Morphologie, vor allem mit Nomen, Präpositionen und Verben, etwas weniger aber mit Artikeln und Adjektiven. Da sie noch relativ niedrige Sprachkompetenzen haben, stellt für sie schon die Konjugation der Verben im Präsens ein Problem dar. Die Lernenden, die die analysierten Texte geschrieben haben, kennen auf ihrer Stufe meist nur das Präsens sowie die Verben sein und haben im Präteritum. Die Lernenden, die über die Themen „Mein Urlaub“ und „Meine Lern- und Sprachbiografie“ 
geschrieben haben, kennen auch das Perfekt. Deshalb ist der Unterschied zwischen der Anzahl der Fehler im Präsens und im Perfekt statistisch nicht vergleichbar.

Tabelle 4: Fehler bei der Verwendung der Verben

\begin{tabular}{|l|l|}
\hline Fehler bei der Verwendung der Verben & $\mathbf{6 1}$ \\
\hline Falsche Konjugation des Verbs im Präsens (oder Präteritum) & 18 \\
\hline Probleme mit dem Präfix der trennbaren Verben & 4 \\
\hline Falscher Tempus & 2 \\
\hline Falsche Form des Partizips im Perfekt & 4 \\
\hline Partizip Perfekt an falscher Stelle & 6 \\
\hline Falsche Form des Vollverbs in Verbindung mit Modalverb & 2 \\
\hline Falsches Hilfsverb im Perfekt & 14 \\
\hline Hilfsverb im Perfekt fehlt & 9 \\
\hline Reflexivpronomen fehlt & 2 \\
\hline
\end{tabular}

Die Fehler bei der Konjugation der Verben beziehen sich auf die Endungen der Verben im Präsens (nur in einem Fall auf das Verb sein im Präteritum), z.B.:

*Dann habe wir ein meeting mit der management. (03)

*Ich arbeitet von 7.00 bis 14. Uhr. (05)

Eine Problematik, die sich auf die Konjugation der Verben im Präsens bezieht, ist auch der Vokalwechsel in der zweiten und dritten Person im Singular. Es wurde ein Beispiel gefunden, wo der Vokalwechsel fälschlicherweise auf die erste Person übertragen wurde:

*Am Morgen ich fänge um 7 Uhr an. (01)

In den Anfängergruppen wird auch die Bildung der Sätze mit trennbaren Verben intensiv geübt. Die typischen Fehler, die diese Verben betreffen, sind die falsche Position der abgetrennten Vorsilbe im Satz, ihr Fehlen, oder die falsche Einstufung eines Verbs als eines trennbaren, z. B.:

*Meinen normalen Arbeitstag fängt um 8 Uhr 30, wann komme ich im Büro. (03) (statt anfangen)

*Um 6.30 Uhr gehe ich zu Fuß zum Arbeit aus. (05) (statt gehen)

*Ich stehn auf am 7.00 uhr. (06) 
Nur in 4 Fällen wurde eine falsche Form des Verbs im Partizip Perfekt verwendet. In zwei Fällen handelt es sich um regelmäßige und in zwei Fällen um unregelmäßige Verben; hier sei nur je ein Beispiel gennant:

*Um 8 Uhr habe ich Spaziergang gemachen. (23)

*Wir haben um sieben Uhr aufgestehen. (28)

In 6 Fällen wurde das Partizip Perfekt statt ans Satzende auf andere Positionen im Satz gestellt, also folgt dem Partizip noch ein Satzglied, das ihm vorangestellt werden müsste, z.B. *Nach der Landung bin ich zum Hotel gefahren mit einem Taxi. (20) Alle solchen Fälle stammen von ein- und demselben Autor ( 3 im Text 20 und 3 im Text 40). Aus den Selbstkorrekturen des Autors (durchstrichenen Wörtern) lässt sich schließen, dass er seine Sätze mehrmals korrigierte und verlängerte - da er sich noch an eine zu erwähnende Information erinnerte, strich er manchmal den Punkt und stellte die zusätzliche Information ans Satzende. Er vergaß aber manchmal, das Partizip ans Ende zu schreiben. Diese Hypothese bestätigte sich auch im Gespräch mit dem betreffenden Lernenden.

In Gruppen, in denen Perfekt schon gelernt wurde, kommt es zur Verwirrung, wann am Satzende das Partizip Perfekt und wann der Infinitiv steht. Da Perfekt intensiv geübt wird, generalisierten die Lernenden in 2 Fällen die Partizipform auch auf Sätze mit Modalverb, z. B.: *Ich mochte Französisch gelernt. (37)

Problematischer als das Partizip ist der andere Teil des Perfekts, nämlich das Hilfsverb. In 14 Fällen wurde das falsche Hilfsverb verwendet und in 9 Fällen fehlt es. Da im Slowenischen nur ein Verb für die Bildung der Vergangenheitsform gebraucht wird (biti - sein), vergessen die Lernenden manchmal, dass sie im Deutschen entweder haben oder sein verwenden sollen. Meistens wird das Hilfsverb haben statt sein verwendet, da haben als erstes gelernt wird. So entstehen Beispiele wie *Am Morgen haben wir nach Hause gefahren. (30)

Wo das Hilfsverb fehlt, geht es um Beispiele, bei denen ein Hilfsverb im Satz geschrieben wurde, das zweite aber fehlt, da die Lernenden annehmen, dass sich ein Hilfsverb auf die beiden Vollverben bezieht. Im folgenden Beispiel hat die Autorin z.B. angenommen, dass haben für beide Verben ausreicht, obwohl das erste Vollverb das Hilfsverb sein und das zweite Vollverb das Hilfsverb haben verlangt: *Am Abend haben wir zum Konzert gegangen und Bier getrunken. (23)

Reflexivverben werden auf dieser Stufe noch nicht gelernt. Trotzdem versuchen die Lernenden sie zu verwenden und vergessen dann manchmal das Reflexivpronomen. Im Korpus sind 2 solche Beispiele zu finden, z. B.: *Wir haben getroffen und Bier getrunken. (23) (statt uns getroffen).

Bei der Verwendung von Nomen wurden mehr Fehler als bei Verben gemacht; diese sind jedoch einheitlicher. Meistens geht es darum, dass vor dem Substantiv die falsche Form des Artikels geschrieben wurde, also steht das Wort im falschen Fall oder wurde 
ihm das falsche Genus zugeschrieben. Diese zwei grammatischen Kategorien scheinen den Lernenden die meisten Probleme zu bereiten, da sie sich anders als im Slowenischen verhalten (bei der Deklination ändert sich der Artikel und nicht die Wortendung; die Genera der jeweiligen Nomen sind in beiden Sprachen nicht gleich).

Tabelle 5: Fehler bei der Verwendung von Nomen

\begin{tabular}{|l|l|}
\hline Fehler bei der Verwendung von Nomen & $\mathbf{8 6}$ \\
\hline Falsches Genus & 31 \\
\hline Falscher Fall & 37 \\
\hline Falsche Pluralbildung & 11 \\
\hline Singular statt Plural & 7 \\
\hline
\end{tabular}

Die Fehler bei der Zuschreibung des Genus kommen sowohl bei den bestimmten (14 Fälle) und unbestimmten ( 5 Fälle) Artikeln als auch bei der Verwendung von Possessivpronomen vor. Hier seien nur einige beispielhafte Sätze zitiert:

*Der Stadt is sehr schön. (02)

*Um 7:00 habe ich eine Caffe. (04)

*Meine arbeitestag ist von 6:30 bis 15.30 Uhr. (04)

Ähnlich sehen die Beispiele aus, bei denen der falsche Fall verwendet wurde: ${ }^{4}$

*Ich bin zusammen mit meine Freundin im Büro. (09)

*Ich arbeite ins Büro. (12)

*OK, dann können wir ein Termin am Dienstag um 9 Uhr reservieren. (17)

Bei dieser Klassifikation ist es fragwürdig, ob die Trennung zwischen Fehlern im Genus und Fehlern im Kasus sinnvoll ist, da erstens die Bestimmung der Fehlerursachen oft nicht möglich ist (beim obigen Beispiel mit dem Wort Termin könnte sich darum handeln, dass die Autorin nicht weiß, dass das Wort männlich ist, oder aber, dass das Wort im Akkusativ stehen müsste) und zweitens, da die Lernenden an diese Unterscheidung bei der Verwendung der Sprache schlichtweg nicht denken. Sie konzentrieren sich oft auf die lexikalische Ebene und geben nicht so viel Betonung auf die Verwendung der Artikel. Deshalb könnten diese beiden Kategorien als eine betrachtet werden.

Die andere Ursache der Fehler ist die Verwendung von Plural: Die Lernenden bilden manchmal die Pluralformen mit falschen Endungen oder ohne Umlate, z.B. *zwei

4 Die Lernenden kennen auf ihrer Stufe nur den Nominativ, Dativ und Akkusativ; den Genitiv haben sie noch nicht gelernt. 
Bucher (28), *viele Sehenswürdigkeiter (29). Wo Plural fälschlicherweise mit der Endung $-s$ gebildet wird, kann angenommen werden, dass es sich um die Interferenz mit dem Englischen handelt, wie im Fall *Analyses (09).

In 7 Fällen wird statt der Pluralform einfach die Singularform verwendet, wie bei den Beispielen *viele lokale Spezialität (20), *zwei Woche (25).

Die Fälle, bei denen der falsche Artikel verwendet wird und dies das falsche Genus oder den falschen Fall des darauffolgenden Nomens verursacht, wurden schon oben besprochen. Hier möchte ich noch an zwei Phänomene aufmerksam machen, die sich nur auf Artikel beziehen. In 33 Fällen fehlt vor dem Nomen ein Wort - meistens ein Artikel, in einigen Fällen würde auch ein Possessivpronomen reichen. Die vergessenen Artikel lassen sich einfach erklären, da das Slowenische keine Artikel kennt und die Lernenden nicht gewöhnt sind, sie zu verwenden. Einige typische Beispiele für diese Problematik wären:

*Ich bekomme neue Arbeit. (08)

*Für den Urlaub war ich auf Insel Zanzibar. (20)

*Kinder haben jeden Abend Eis gewollt. (28)

Die restlichen 3 Fehlerbeispiele, die sich auf den Einsatz der Artikel beziehen, sind Fälle, in denen ein bestimmter Artikel statt eines unbestimmten verwendet wurde.

Bei Adjektiven geht es um die falschen Numerus-, Genus- und Kasusendungen, wobei in 11 Fällen keine Endung ausgewählt wurde und das Adjektiv einfach in seiner unflektierten Form eingesetzt wurde, in 11 Fällen wurde aber eine Endung geschrieben, die nicht mit dem darauffolgenden Nomen übereinstimmt.

*Ich mag meine neu arbeit. (04)

*Ich mog die Deutsch Sprache, weil ich gerne deutsche Musik gehört habe. (34)

*Da habe ich Italienisch gelernt, weil es bilingual Gebiet ist. (39)

*Dann musste ich eine neue Job gefindet, weil mein studentstatus abgelaufen ist. (40)

Diese Fehler sind meines Erachtens auf der Stufe A1 und am Anfang der Stufe A2 nicht schwerwiegend, da die Lernenden noch nicht alle Regeln für die Adjektivdeklination gelernt haben. Sie beeinträchtigen keinesfalls die Verständlichkeit der Texte.

Von den 37 Fehlern, die sich auf Präpositionen beziehen, sind 33 solche, bei denen eine falsche Präposition ausgewählt wurde, und 4 solche, bei denen die Präposition fehlt. Manchmal wiederholen sich diese Fehler und werden für einen bestimmten Lernenden (im Text 06 wurde fünfmal am statt $u m$ in Verbindung mit einer Zeitangabe verwendet: *Ich habe pause am 14.00 uhr. (06)) oder für eine Gruppe typisch (Texte 13, 14, 15 stammen aus derselben Gruppe und verwenden typischerweise nach für das Wiedergeben 
einer Richtung, in die man sich bewegt: *Ich fahre mit mein Auto nach Bahnhof in Škofja Loka. (14)) Die Regeln für den Einsatz von Präpositionen, vor allem in Verbindung mit Zeitangaben, werden auf der Stufe A1 intensiv geübt, trotzdem kommt es aber zu Fehlern.

\subsection{Syntaktische Fehler}

Die meisten Fehler auf der Ebene der Syntax beziehen sich auf die Wortstellung im Satz. Die Lernenden vergessen besonders oft, dass das Verb im Hauptsatz an der zweiten Stelle steht. Von 22 solchen Fällen stammen 8 aus dem Text 13. Zumeist wird am Satzanfang eine Zeitangabe geschrieben, worauf dann das Subjekt statt des Verbs folgt.

*Am Montag oder am Dienstag ich schreibe meine Deutsch Hausaufgabe. (13)

*Am Donnerstag wir besuchen den Tanzkurs. (13)

Über Nebensätze haben nur die AutorInnen der Texte zum Thema Meine Lern- und Sprachbiografie gelernt, und zwar nur über Nebensätze mit der Konjunktion weil. Trotzdem versuchen die Lernenden, Nebensätze zu bilden. Da sie aber noch nicht mit den Regeln für die Wortstellung im Nebensatz vertraut sind, schreiben sie z. B.:

*Ich mag meinen neuen Job, aber ich mag nicht meinen Arbeitszeit weil ich arbeite auch am Wochenende. (01)

*Schöne Gruße aus Schweden wo habe ich dem Urlaub gemacht! (22)

*Ich spreche auch gut Kroatisch, weil meine Großmutter ist aus Kroatien gekommen. (33)

Es ist wichtig zu erwähnen, dass alle angeführten Sätze von AutorInnen stammen, die für ihre Stufe relativ viel verstehen und gut sprechen können - sie versuchen eine anspruchsvollere Struktur zu verwenden, um die logischen Verbindungen zwischen den Informationen auszudrücken, weil ihnen die einfachen Hauptsätze nicht mehr genügen. Aus den angeführten Sätzen ist es ersichtlich, dass das Verb entweder direkt nach dem ersten Satzglied (meist Subjekt) wie im Hauptsatz stent, wie die Fälle aus den Texten 01 und 33 zeigen, oder aber direkt nach der Konjunktion, wie im Fall aus dem Text 22.

Über fehlende Artikel, Hilfsverben und Präpositionen wurde schon oben berichtet; auf die Ebene der Syntax gehören aber Beispiele, wo ein ganzes Satzglied fehlt. In 5 Fällen ist das ein Subjekt (dreimal ich, einmal $d u$, einmal es) und in einem Fall fehlt das Objekt. Die Fehler, bei denen das Pronomen in der Rolle des Subjekts fehlt, sind meines Erachtens eine Folge der Interferenz mit dem Slowenischen, da das Slowenische nicht 
verlangt, dass das Subjekt immer explizit mit einem Personalpronomen genannt wird. Hier sei nur ein Beispielsatz für diese Problematik zitiert:

*Dann putze meine zehne. (11)

Manchmal wird ein Wort zu viel geschrieben, und zwar ist das am häufigsten (8 Fälle) eine Präposition. Die anderen syntaktischen Fehler sind unterschiedlich, sie beziehen sich auf unvollständige Sätze (bei denen mehr als ein Satzglied fehlt), unlogische Konstruktionen und unlogische Verbindungen zwischen den Informationen.

\subsection{Lexikalische und semantische Fehler}

Die häufigsten Fehler auf lexikalischer bzw. semantischer Ebene sind die falsch verwendeten Wörter. Manchmal zeigen diese Beispiele einfach nur, dass die Lernenden strategisch das Wissen einsetzen, über das sie verfügen, um eine neue Information auszudrücken, z. B.:

*Um 22.00 Uhr sehe ich Berichte fern. (15) (statt die Nachrichten im Fernsehen)

*Ich bin sehr gut. (26) (statt es geht mir sehr gut)

*Wir hätten Flugtickets sehr billig gekauft. Nur 40 eur für beide Wege. (31) (statt in beide Richtungen / hin und zurück)

Oft aber helfen sich die Lernenden mit dem Wissen aus anderen Sprachen. Es kommt zu Interferenzfehlern aufgrund von falschen Freunden, vor allem mit dem Englischen. In 5 Beispielen wurde das Verb haben in Verbindung mit Essen/Trinken nach dem Muster von to have coffee, to have breakfast eingesetzt:

*Um 7:00 habe ich eine Caffe. (statt trinke)

*Ich stehe um sechs Uhr auf und ich habe Früstuck. (12) (statt frühstücke)

Einige Beispiele anderer Interferenzfehler wären:

*Es [ein Schiff] ist sehr gut preserviert ins museum. (22) (statt erhalten nach dem englischen preserve)

*Ich habe die Grundschule ins Koper gemacht. (39) (statt besucht/abgeschlossen nach dem slowenischen opravil/naredil šolo)

Oft wird aber nicht ein deutscher falscher Freund verwendet, sondern einfach ein Wort aus einer anderen Sprache eingesetzt, das im Deutschen überhaupt keine Bedeutung hat: 
*Mein freund und Ich hat mit helicopter gefahren. (21) (statt Hubschrauber nach slow. helikopter / engl. helicopter)

*Auch English und German ich brauche für mein beruf. (35) (statt Deutsch nach engl. German)

*Ich möchte mit mein frienden aus Deutsch und Östreich sprechen. (35) (statt Freunden nach engl. friend)

Da die Lernenden über die Informationen oft nur isoliert nachdenken und sehr lange brauchen, um einen Satz zu formulieren, kommt es auch dazu, dass sie sich mit Pronomen auf die falsche Person beziehen. Manchmal führt dies auch dazu, dass statt Siezen das Duzen verwendet wird oder umgekehrt.

\subsection{Fehler auf der Ebene des gesamten Textes}

Hier geht es nur um das Fehlen der Anrede und der Grußformel in den Texten, wo die Form eines Briefes/einer Postkarte/einer E-Mail verlangt war. Die Lernenden schreiben isolierte Sätze, die sich nicht aufeinander beziehen; in einigen Beispielen schreiben sie jeden Satz in eine neue Zeile und im Text 11 hat die Autorin sogar Stichpunkte für jeden Absatz gezeichnet, die den einzelnen Fragen in der Aufgabenstellung entsprechen. Die logische Verbindung der Informationen ist für die Lernenden auf dieser Stufe schwer, gleich wie die Einhaltung der Textform und der pragmatischen Normen (Duzen/Siezen). Dies verbindet sich mit der obigen Feststellung, dass Nebensätze öfters von den anspruchsvolleren Lernenden geschrieben werden, da diese öfters versuchen, die Informationen aneinander zu knüpfen. Bei ihnen passiert nicht, dass jeder Satz in einer neuen Zeile angefangen wird oder dass die Texte nur aus isolierten Einzelinformationen bestehen.

Die Analyse hat gezeigt, dass den Lernenden die deutsche Rechtschreibung und Morphologie die meisten Probleme bereiten. Auf der Ebene der Rechtschreibung sind die Großschreibung der Nomen und das richtige Buchstabieren der Wörter hervorzuheben. Auf diese Lernschwierigkeiten kann gezielt eingegangen werden - eine nützliche Unterrichtsmethode, bei der die Lernenden auf die Rechtschreibung aufpassen müssen, ist das Diktat. Es empfiehlt sich, nach dem Schreiben des Diktats die Lernenden ihre Texte mit dem Nachbarn/der Nachbarin austauschen zu lassen, damit jeder bzw. jede den Text des Kollegen/der Kollegin korrigiert und sich dabei noch einmal auf die Rechtschreibung konzentriert. Eine andere Aufgabe, die auf die Rechtschreibkompetenz zielt, ist das Lösen der Kreuzworträtsel, bei denen alle Buchstaben richtig gesetzt werden müssen. 
Auf der Ebene der Morphologie scheint der schwierigste Bereich die Setzung der richtigen Artikel zu sein, also die Signalisierung des Genus und Kasus der Substantive. Die Lernenden schreiben entweder die falschen Artikel oder lassen sie aus, da das Slowenische keine Artikel kennt. Oft wird auch eine falsche lokale oder temporale Präposition verwendet. Bei den Verben entstehen die meisten Fehler im Bereich der Konjugation im Präsens und bei der Auswahl des richtigen Hilfsverbs im Perfekt. Die Fehler auf morphologischer Ebene lassen sich mithilfe der Einsetzübungen beheben, wo die Lernenden über die korrekte Form im gegebenen Satz nachdenken müssen oder von den angebotenen Formen die richtige auswählen.

Eine wichtige Fehlerquelle ist auch die falsche Lexik. Die Interferenzen zwischen dem Deutschen und den anderen Sprachen sind gut an den Fehlern erkennbar, die aufgrund der falschen Freunde entstehen. Hier ist es wichtig, die Lernenden auf die Unterschiede und falsche Freunde aufmerksam zu machen, damit sie sich die richtigen Formen einprägen.

Die Fehleranalyse verdeutlicht auch, wie bestimmte Fehler nur oder hauptsächlich von ein- und demselben Lernenden immer wieder gemacht werden (obwohl sie schon einmal korrigiert wurden).

Es empfiehlt sich, den Lernenden ausgewählte Sätze mit typischen Fehlern aus eigenen Aufsätzen zu zeigen und sie die Fehler auf allen sprachlichen Ebenen darin identifizieren und korrigieren zu lassen. Dadurch werden sie auf die typischen Fehler aufmerksam und werden sie bei den künftigen Aufgaben vielleicht selber schon während des Schreibens korrigieren können. Selbstverständlich müssen dann die korrekte und die inkorrekte Form verglichen werden und die Lehrperson (oder eine/einer von den besseren Lernenden) muss erklären, warum die eine Version besser ist als die andere. Sinnvoll in diesem Sinne wäre es auch, die Lernenden den korrigierten Aufsatz noch einmal abschreiben zu lassen, damit sie nicht nur über die Korrekturen drüber fliegen und sie sich nicht einprägen.

\section{BIBLIOGRAPHIE}

BUßMANN, Hadumod (1990) Lexikon der Sprachwissenschaft. 2., völlig neu bearbeitete Auflage. Stuttgart: Kröner.

ČOK, Lucija (1999) Medjezikovni transfer in zavest o jeziku. L. Čok et al. (Hrsg.), Učenje in poučevanje tujega jezika. Smernice za učitelje $v$ drugem triletju osnovne šole. Ljubljana: Pedagoška fakulteta/Koper: Znanstveno-raziskovalno središče, 49-52.

Gemeinsamer europäischer Referenzrahmen für Sprachen (2013) Hrsg. vom Goethe-Institut, der Ständigen Konferenz der Kulturminister der Länder in der Bundesrepublik Deutschland (KMK) et al. München: Klett Langenscheidt (zitiert als GER). 
KLEPPIN, Karin (1998) Fehler und Fehlerkorrektur. Fernstudieneinheit 19. Berlin: Langenscheidt.

MLAKAR GRAČNER, Doris (2018) Fehler beim schriftlichen Produzieren von Texten im DAF-Studium. Eine Untersuchung am Beispiel slowenischer Germanistikstudierender. Vestnik za tuje jezike 10 (1), 219-237.

MUSTER, Ana Marija (2008) Jezikovne napake pri učenju nemščine. J. Skela (Hrsg.), Učenje in poučevanje tujih jezikov na Slovenskem: Pregled sodobne teorije in prakse. Ljubljana: Tangram, 604-617.

MUSTER, Ana Marija (2000a) Napake slovenskih učencev pri učenju nemščine. Ljubljana: Zavod Republike Slovenije za šolstvo.

MUSTER, Ana Marija (2000b) Was meinen die slowenischen Deutschlehrerinnen über Fehler? Vestnik Društva za tuje jezike in književnosti Slovenije XXXIV, 477-486.

\section{POVZETEK}

\section{Analiza napak v besedilih učencev nemščine na začetni stopnji}

Prispevek analizira tipične napake v pisnih izdelkih (domačih in izpitnih nalogah) odraslih, katerih prvi jezik je slovenščina in ki obiskujejo tečaj nemščine na začetni stopnji. V teoretičnem delu so orisani pojem napake v okviru poučevanja tujih jezikov, klasifikacija napak po jezikovnih ravninah od pravopisa do pragmatike in tipični vzroki za napake pri učenju tujega jezika, pri čemer je izpostavljen pojem interference. Temu sledita klasifikacija in opis napak iz zbranega korpusa 40 besedil. Rezultati analize pokažejo, da učenci največ napak pri pisanju delajo na ravni pravopisa in oblikoslovja. Pri pravopisu izstopata pisanje samostalnikov z veliko začetnico in pravilno črkovanje besed, pri oblikoslovju pa spol in sklon samostalnikov ter spreganje glagolov. Relativno pogoste so tudi napake $\mathrm{v}$ zvezi z rabo neustreznih predlogov in neuporabo določnih in nedoločnih členov. V napačni rabi leksike se kažeta vpliva slovenščine kot prvega in angleščine kot tujega jezika, ki ju učenci že poznajo. Glavni vzroki za napake so znotrajjezikovna in medjezikovna interferenca ter nepoznavanje pravil $\mathrm{v}$ tujem jeziku. $\mathrm{V}$ zadnjem delu prispevka je opisanih nekaj vaj, ki naj bi učencem pomagale pri izogibanju tipičnim napakam; poudarek je na ozaveščanju in iskanju lastnih napak.

Ključne besede: napake, analiza napak, vzroki za napake, interferenca, stopnja A1 SEJO 


\section{ABSTRACT}

\section{Analysis of errors in the texts of German learners at a beginner level}

The paper analyses the typical errors and mistakes in texts (homeworks and exams) written by adults whose first language is Slovene and who are learning German at a beginner level. The theoretical part describes the concept of a mistake in the framework of language education, the classification of mistakes according to the language levels from the orthography to the pragmatics, and the most common causes of mistakes in the process of language acquisition; the concept of interference is explained in this part. This is followed by a classification and description of mistakes from the collected corpus of 40 texts. The results of the analysis show that learners make the most mistakes on the levels of orthography and morphology. The most problematic are the capitalization of nouns and the correct spelling on the level of orthography, and the gender and case of nouns as well as the conjugation of verbs on the level of morphology. Mistakes caused by the incorrect use of prepositions and the lack of definite and indefinite articles are also relatively common. The incorrect use of vocabulary shows the influence of Slovene as the first language and English as the foreign language already known to the students. The most important causes of mistakes are the intralingual and interlingual interferences, as well as the lack of knowledge of the rules in the foreign language. In the last part of the paper, some exercises are described that are intended to help learners avoid common mistakes, and emphasis is put on the awareness of one's own mistakes.

Keywords: errors, error analysis, causes of errors, interference, level A1 of CEFR 